ISSN 0103-9954

\title{
CARACTERÍSTICAS QUÍMICAS DE UM LATOSSOLO VERMELHO EM RECUPERAÇÃO SOB PLANTIO DE Pinus spp.
}

\author{
CHEMICAL CHARACTERISTICS OF A RED LATOSOL IN RECOVERING UNDER Pinus spp.
}

\author{
Alexandre Marques Da Silva ${ }^{1}$ Daniela Sílvia de Oliveira Canuto ${ }^{2}$ Marlene Cristina Alves ${ }^{3}$ \\ Salatiér Buzetti ${ }^{4}$ Mario Luiz Teixeira de Moraes ${ }^{5}$ Arnaldo Yoso Sakamoto ${ }^{6}$
}

\begin{abstract}
RESUMO
Para a recuperação de um solo é preciso selecionar e identificar espécies aptas às novas condições edáficas e que, de forma rápida, acelerem a estruturação e formação dos horizontes mais superficiais do solo. $\mathrm{O}$ experimento teve como objetivos avaliar o desenvolvimento de algumas espécies e variedades de Pinus, visando à detecção daquelas que apresentam maior potencialidade de adaptação e recuperação do solo degradado na região de cerrado e, para isto, foram avaliadas as condições químicas do solo em que se encontram as mesmas. Aos 20 anos, após o plantio, analisaram-se as características silviculturais e as propriedades químicas do solo. Foram coletadas em cada parcela amostras deformadas nas camadas de 0,0-0,10;0,10-0,20; 0,20-0,40 e 0,40-0,60 m. A utilização de espécies de Pinus na recuperação de áreas degradadas mostrou-se promissora melhorando as condições químicas do solo na camada de $0,00-0,10 \mathrm{~m}$, destacando-se o Pinus caribaea var. hondurensis por apresentar bom crescimento aliado a sua sobrevivência, o que garante a cobertura do solo devido à deposição de matéria orgânica aumentando a soma de bases e a capacidade de troca catiônica. As espécies de Pinus estão proporcionando condições para o aumento da diversidade de espécies espontâneas.
\end{abstract}

Palavras-chave: solos degradados; qualidade do solo; área de empréstimo.

\section{ABSTRACT}

For the recovery of a soil, it is necessary to select and to identify suitable species to new soil conditions and, quickly, accelerate the structuring and formation of more superficial horizons of the soil. The experiment aimed to evaluate the development of some species and varieties of Pinus, aiming at detecting those that present the greatest potential for adaptation and recovery of degraded land in the savannah region, and for this, we evaluated the chemical conditions of the soil where they are present. At 20 years after planting, we analyzed the silvicultural characteritics and the chemical properties of the soil. In each plot, deformed

1 Geógrafo, Doutorando, Técnico Agropecuário do Departamento de Fitotecnia, Tecnologia de Alimentos e Sócio Economia, Universidade Estadual Paulista (UNESP), Faculdade de Engenharia Ilha Solteira, Av. Brasil 56, Centro, CEP 15385-000, Ilha Solteira (SP), Brasil. amsilva@agr.feis.unesp.br

2 Bióloga, Dra ${ }^{\mathrm{a}}$, Professora Colaborador do Programa de Pós-Graduação em Agronomia, Universidade Estadual Paulista (UNESP), Faculdade de Engenharia Ilha Solteira, Av. Brasil 56, Centro, CEP 15385-000, Ilha Solteira (SP), Brasil.dsocanuto@gmail.com

3 Engenheira Agrônoma, Dr ${ }^{\mathrm{a}}$., Professora Titular do Departamento de Fitossanidade, Engenharia Rural e Solos, Universidade Estadual Paulista (UNESP), Faculdade de Engenharia Ilha Solteira, Av. Brasil 56, Centro, CEP 15385-000, Ilha Solteira (SP), Brasil. mcalves@agr.feis.unesp.br

4 Engenheiro Agrônomo, Dr., Professor Titular do Departamento de Fitossanidade, Engenharia Rural e Solos, Universidade Estadual Paulista (UNESP), Faculdade de Engenharia Ilha Solteira, Av. Brasil 56, Centro, CEP 15385-000, Ilha Solteira (SP), Brasil. sbuzetti@agr.feis.unesp.br

5 Engenheiro Agrônomo, Dr., Professor Titular do Departamento de Fitotecnia, Tecnologia de Alimentos e Sócio Economia Universidade Estadual Paulista (UNESP), Faculdade de Engenharia Ilha Solteira, Av. Brasil 56, Centro, CEP 15385-000, Ilha Solteira (SP), Brasil. teixeira@agr.feis.unesp.br

6 Geógrafo, Dr., Professor do Departamento de Ciências Humanas, Universidade Federal de Mato Grosso do Sul, Av. Ranulfo Marques Leal 3484, Distrito Industrial, CEP 79610-000, Três Lagoas (MS), Brasil. arnaldo. sakamoto@ufms.br

Recebido para publicação em 10/03/2011 e aceito em 13/04/2015 
samples were collected in the layers of $0.0-0.10,0.10-0.20,0.20-0.40$ and $0.40-0.60 \mathrm{~m}$. The use of Pinus species in reclamation showed promising improving the chemical conditions of the soil layer from 0.00 to $0.10 \mathrm{~m}$, highlighting the Pinus caribaea var. hondurensis to present a good growth combined their survival, which guarantees the coverage of the soil due to deposition of organic matter by increasing the sum of bases and cation exchange capacity. The species of Pinus are providing conditions for the increasing of the diversity of spontaneous species.

Keywords: degraded soils, soil quality; lending area.

\section{INTRODUÇÃO}

Durante muito tempo falou-se que a geração de energia hidrelétrica não causava impactos ambientais negativos ao meio ambiente, no entanto, muitas pesquisas demonstraram que este tipo de geração de energia antes tido como "energia limpa" causa sérios impactos ambientais negativos desde a sua construção até o seu funcionamento (INATOMI; UDAETA, 2011). Um dos principais danos ambientais gerados pela construção de hidrelétricas são as áreas de empréstimos, locais utilizados para a remoção de solo e descarte de materiais diversos, causando modificação da paisagem, e produzindo impactos ambientais, sociais e econômicos.

Área de empréstimo é o local do qual se retira material mineral (argila, rochas, etc.), destinado às obras de engenharia. As áreas de empréstimo são definidas após prospecções geológicas, considerando fatores técnicos (mecânica dos solos) e econômicos (distância de transporte e volume da jazida), compatibilizados com fatores ecológicos e estéticos (MÜLLER, 1995). As áreas de empréstimo apresentam baixo índice de revegetação natural, ficando seus solos desnudos e expostos aos processos de erosão acelerada, sobretudo os ocasionados pelas chuvas, pois possui baixa taxa de infiltração e armazenamento de água, por possuir um solo compactado pela movimentação de máquinas pesadas ou pela exposição do subsolo, não fornecendo condições adequadas para o desenvolvimento de plantas, pois apresenta baixos teores de matéria orgânica, deficiência de oxigênio e aumento da densidade do solo, dificultando a penetração de raízes. No momento que a cobertura vegetal nativa é retirada de uma região e substituída por uma cidade, outra cultura, pasto, etc., a dinâmica de evolução do relevo altera-se. É o momento de ruptura de um processo que foi elaborado ao longo das eras, e da perda da estabilidade delicada dos elementos da relação. A evolução das formas sai da escala geológica para entrar na história. Apesar dos poucos estudos a este respeito, poderia se afirmar que o relevo desprovido de sua vegetação natural evoluirá para formas diferentes (e, sem dúvida, muito mais rapidamente) daquelas que evoluiria em condições naturais (NOFFS, 1982).

As áreas de empréstimo constituem-se em um ecossistema degradado, que teve eliminado, juntamente com a vegetação, os seus meios de regeneração bióticos como o banco de sementes, banco de plântulas, chuva de sementes e rebrota. Apresenta, portanto, baixa resiliência, isto é, seu retorno ao estado anterior pode não ocorrer ou ser extremamente lento. Com estas características edáficas, a escolha das espécies que iniciarão a sucessão ecológica é de suma importância, tendo que ser capaz de produzir condições favoráveis para que, gradualmente sejam substituídas por outras espécies até que as condições ambientais cheguem a uma diversidade compatível com as características daquele ambiente. A adaptação e desenvolvimento dessas espécies dependerá das condições físicas, químicas, biológicas e hídricas do solo, bem como das condições do microclima local (ALVES, 2001).

$\mathrm{O}$ gênero Pinus ocorre naturalmente em várias regiões do mundo em diferentes climas e solos. Segundo Gurgel Filho (1965-66), a ocorrência natural de Pinus acha-se entre $2^{\circ} 06^{\prime}$ de latitude sul ou centro de Sumatra, até $69^{\circ} 45^{\prime}$ de latitude norte, ou seja, na Finlândia, Mongólia e Sibéria. A maior quantidade de espécies em estado nativo encontra-se no Hemisfério Norte. Quanto à altitude, encontramse desde quase ao nível do mar até cerca de 3000 $\mathrm{m}$. A grande maioria está em planaltos ou regiões montanhosas com cerca de $700 \mathrm{~m}$.

Silva et al. (2002a), trabalhando em área degradada com diferentes espécies de Pinus com 5 anos, e manejo de um Latossolo em Selvíria MS, concluíram que o gênero Pinus mostrou-se promissor para a recuperação destas áreas, devido ao bom desenvolvimento e sobrevivência que garante uma razoável cobertura do solo, destacando-se $o$ 
Pinus oocarpa, Pinus patula var. tecunumanii e Pinus caribaea var bahamensis.

Para a revegetação de áreas degradadas não existe uma receita de manejo, portanto, para cada situação o maior número de espécies nativas ou exóticas deve ser testada, considerando que, no caso de exóticas, deve-se conhecer o comportamento da espécie em termos de sucessão, para evitar que estas passem a ser dominantes no ecossistema. Dessa forma, objetiva-se uma diversidade razoável de espécies adaptadas às difíceis condições iniciais (CAMPELLO, 1998).

Silva et al. (2011) e Silva et al. (2012) avaliaram as características químicas de Latossolo Vermelho distrófico sob reflorestamento ciliar após 20 anos de plantio em área de cerrado e concluíram que as espécies plantadas estão contribuindo com a deposição de material orgânico suficiente para que haja reciclagem de nutrientes, mantendo as características químicas do solo em boas condições para que ocorra o estabelecimento da mata ciliar.

Os Planos de Recuperação de Áreas Degradadas (Prad), apesar de exigidos desde 1989, são relativamente recentes nos empreendimentos e há uma evidente dissociação entre as medidas praticadas e aquelas preconizadas nesses planos. A maior parte dos trabalhos de recuperação de áreas degradadas pela mineração que se tem registrado no Estado de São Paulo tem caráter incipiente e se baseia especialmente na execução de medidas restritas de revegetação, visando atenuar o impacto visual gerado (MECHI; SANCHES, 2010).

Neste sentido, o presente trabalho teve como objetivos avaliar o desenvolvimento de algumas espécies e variedades de Pinus, visando à detecção daquelas que apresentam maior potencialidade de adaptação e recuperação ao solo degradado na região de Cerrado, e avaliar as condições químicas do solo em que as mesmas se encontram.

\section{MATERIAL E MÉTODOS}

O experimento foi instalado em 01 de março de 1982 em uma área de empréstimo localizada na Fazenda de Ensino, Pesquisa e Extensão, pertencente à Faculdade de Engenharia, Campus de Ilha Solteira (FE/Unesp), área de Produção Animal, localizada no município de Selvíria, Estado de Mato Grosso do Sul situada a $51^{\circ} 23^{\prime} 59,09^{\prime \prime}$ de longitude Oeste de Greenwich e $20^{\circ} 22^{\prime} 03,97^{\prime \prime}$ de latitude Sul, a uma altitude de $334 \mathrm{~m}$. O tipo climático é Aw, segundo a classificação de Köppen, caracterizado como tropical úmido, com estação chuvosa no verão e seca no inverno (DEMATTÊ, 1980), apresentando temperatura média anual de $25^{\circ} \mathrm{C}$ e precipitação anual de $1.300 \mathrm{~mm}$ (CENTURION, 1982).

Demattê (1980) caracterizou o solo original do local como sendo do tipo Latossolo VermelhoEscuro álico com textura média (20 - 35\% argila), muito profundo, rico em sesquióxido. A sua fração argila é de baixa atividade e denominada essencialmente pela gibbsita e caulinita. $\mathrm{O}$ solo, classificado segundo o Sistema Brasileiro de Classificação de Solos (SANTOS et al., 2013), é um Latossolo Vermelho Distrófico. A área de estudo está localizada no Planalto da Bacia Sedimentar do Paraná, apresenta declives muito suaves, relevo plano e suavemente ondulado (ALVES, 2001). A área estudada possuía vegetação natural de Cerrado. A pesquisa foi desenvolvida no subsolo do solo citado anteriormente, pois houve remoção de uma camada de $8,60 \mathrm{~m}$ de profundidade para o terrapleno e fundação da barragem, com o objetivo de se construir a usina Hidrelétrica de Ilha Solteira - SP.

Foi feita uma única adubação inicial por cova em todo o experimento de $30 \mathrm{~g}$ de $\mathrm{N}, 80 \mathrm{~g}$ de $\mathrm{P}_{2} \mathrm{O}_{5}$ e $15 \mathrm{~g}$ de $\mathrm{K}_{2} \mathrm{O}$, usando como fontes respectivamente o nitrato de amônio, termofosfato yoorin e o cloreto de potássio Não foi feita a correção do $\mathrm{pH}$ do solo no plantio do experimento. Ao longo dos anos do experimento não houve nenhuma outra adubação química, orgânica ou correção do $\mathrm{pH}$ do solo.

$\mathrm{O}$ delineamento experimental utilizado foi de blocos casualizados, constituindo de quatro repetições e seis tratamentos. Os tratamentos são compostos por espécies e variedades de Pinus: Pinus caribaea var. bahamensis; Pinus caribaea var. caribaea; Pinus caribaea var. hondurensis, Pinus oocarpa (Agudos-Brasil); Pinus oocarpa (Guaimaca-Honduras) e Pinus strobus var. chiapensis. Cada parcela possui 42 plantas, sendo 20 úteis, plantadas no espaçamento de 2,5 x 2,0 $\mathrm{m}$. O Pinus strobus var. chiapensis não suportou as condições do local, sendo que todas as plantas morreram, no entanto, estas parcelas vêm sendo utilizadas como testemunha. $\mathrm{O}$ experimento foi analisado, portanto, com apenas cinco tratamentos, para as características silviculturais avaliadas. As mudas das espécies e variedades de Pinus utilizadas no experimento foram provenientes da antiga Cia. Agroflorestal Monte Alegre (CAFMA), atual Duratex, localizada no município de Agudos - SP, sendo produzidas em recipientes do tipo laminado. 
Aos 20 anos após o plantio, analisaramse as características silviculturais sendo: diâmetro a altura do peito (DAP, em $\mathrm{cm}$ ) mensurado a 1,30 $\mathrm{m}$ acima do nível do solo; altura total das árvores (ALT, em m); volume de madeira (VOL, em $\mathrm{m}^{3}$ $\operatorname{arv}^{-1}$ ) e sobrevivência de plantas no campo (SOB, em \%). A forma do fuste das árvores (FOR), obtida com base em escala de notas: (1) tortuosidade acentuada em toda extensão; (2) ligeiramente tortuoso em toda extensão; (3) reto na parte superior e tortuosidade acentuada na base; (4) reto na parte inferior e tortuosidade acentuada no topo; (5) reto com ligeira tortuosidade na base; (6) reto com ligeira tortuosidade na parte superior e (7) perfeitamente reto. Bifurcação da árvore (BIF), obtida por notas: (1) bifurcação no terço inferior do fuste; (2) bifurcação no terço mediano do fuste; (3) bifurcação no terço superior do fuste e (4) fuste sem bifurcação. O caractere rabo de raposa (foxtail) (FT), também obtida por uma escala de notas, baseando-se em Kageyama, Oires e Herrera Prera (1984): (0) ausência de foxtail; (1) internódio com $1,5 \mathrm{~m}$; (2) internódio entre 1,5 a $3,0 \mathrm{~m}$; (3) 3,0 a $4,5 \mathrm{~m}$; (4) 4,5 a $6,0 \mathrm{~m}$; (5) 6,0 a $7,5 \mathrm{~m}$; (6) 7,5 a 9,0 m; (7) 9,0 a $10,5 \mathrm{~m}$; (8) 10,5 a $12,0 \mathrm{~m}$ e (9) 12,0 a 13,5 m. O DAP foi realizado com a utilização de um paquímetro florestal e a altura mensurada com hipsômetro eletrônico Vertex. A sobrevivência foi baseada na percentagem de plantas existentes. O volume foi calculado a partir das medidas de DAP e altura das plantas.

Foi realizado na área experimental um levantamento qualitativo da ocorrência natural de espécies nativas. O levantamento não considerou os tratamentos de Pinus, sendo realizado em área total para verificar quais espécies espontâneas ocorriam na área experimental. As espécies foram identificadas in loco e por meio de literatura especializada. A identificação da nomenclatura botânica com base nos trabalhos de: Lorenzi (2002); Silva Júnior et al. (2005); Lorenzi (2008) e Lorenzi (2009). As espécies foram classificadas em famílias de acordo com o sistema do Angiosperm Phylogeny Group II (2003).

Em janeiro de 2003 foram coletadas quatro amostras simples deformadas nas camadas de 0,0 0,$10 ; 0,10-0,20 ; 0,20-0,40$ e $0,40-0,60 \mathrm{~m}$ em cada parcela, com auxílio de um trado de caneca, com diâmetro interno de $0,08 \mathrm{~m}$ e altura de $0,20 \mathrm{~m}$, constituindo um volume de $1,00510 \mathrm{~m}^{-3}$, as quais, após misturadas, geraram uma amostra composta que foi analisada segundo metodologia descrita em
Raij et al. (2001). O solo foi seco ao ar, processado em moinho de solo com rotor vertical e martelos móveis (Marconi MA330) passado por peneira com malha de $2 \mathrm{~mm}$, sendo as análises químicas realizadas no Laboratório de Análise do Solo e Tecido Vegetal do Departamento de Fitotecnia, Tecnologia de Alimentos e Socioeconomia, Faculdade de Engenharia da Universidade Estadual Paulista "Júlio de Mesquita Filho" (UNESP), Campus de Ilha Solteira.

$\mathrm{O}$ pH foi determinado em solução de $\mathrm{CaCl}_{2} 2 \mathrm{H}_{2} \mathrm{O} \quad 0,01$ mol. $\mathrm{L}^{-1}$ através de potenciometria (pHmetro Luca-210); o teor de $\mathrm{H}$ + Al foi determinado pelo método SMP utilizando potenciometria (pHmetro Luca-210); Al trocável extraído por solução $\mathrm{KCl} 1 \mathrm{~mol} \mathrm{~L}^{-1}$, determinado por titulometria com $\mathrm{NaOH}$ 0,025 mol.L $\mathrm{L}^{-1}$. A matéria orgânica do solo foi determinada por meio do método colorimétrico (solução de $\mathrm{Na}_{2} \mathrm{Cr}_{2} \mathrm{O}_{7}$. $2 \mathrm{H}_{2} \mathrm{O} 0,667 \mathrm{~mol} \mathrm{~L}^{-1}+\mathrm{H}_{2} \mathrm{SO}_{4} 5 \mathrm{~mol} \mathrm{~L}^{-1}$ ), através de espectrofotometria (Biospectro SP-22). Os teores de fósforo, potássio, cálcio e magnésio pelo método de extração com resina trocadora de íons, sendo o $\mathrm{Ca}$ e $\mathrm{Mg}$ determinados por espectrometria de absorção atômica (Varian 55B), K trocável por fotometria de chama (Micronal B462) e P disponível por espectrofotometria (Biospectro SP-22); o enxofre disponível foi extraído por $\mathrm{Ca}\left(\mathrm{H}_{2} \mathrm{PO}_{4}\right)_{2} 0,01$ mol.L $\mathrm{L}^{-1}$, e determinado por turbidimetria (Biospectro SP-22).

Os resultados foram analisados efetuandose a análise de variância e teste de Tukey no nível de $5 \%$ de probabilidade para comparação de médias, sendo utilizado o programa SAS (SAS INSTITUTE, 1990) para análise dos dados.

\section{RESULTADOS E DISCUSSÃO}

Os dados referentes às características silviculturais diâmetro a altura do peito (DAP), altura total (ALT), volume (VOL), forma do fuste das árvores (FOR), foxtail (FT), bifurcação (BIF) e a sobrevivência das plantas (SOB) das 5 espécies estudadas estão apresentados na Tabela 1. Não houve diferenças significativas para altura total, forma do fuste, foxtail e bifurcação. O Pinus oocarpa (Brasil) apresentou as melhores médias para o diâmetro a altura do peito e volume, respectivamente $27,45 \mathrm{~cm}$ e $1,01 \mathrm{~m}^{3}$ arv $^{-1}$, e os Pinus caribaea var. bahamensis e Pinus caribaea var. caribaea apresentaram os menores resultados para diâmetro a altura do peito e volume. O Pinus caribaea var. bahamensis apresentou uma maior adaptação no 
local onde está instalado o experimento com 71,25 $\%$ de sobrevivência e o Pinus oocarpa (Brasil) apresentou $26,25 \%$. Os melhores resultados obtidos de DAP e volume apresentado pela espécie Pinus oocarpa (Brasil) podem estar relacionados com a baixa sobrevivência, pois menor número de plantas por parcela proporciona menor competição por luz e nutriente entre as plantas.

Avaliados aos 18 anos de idade por Silva et al. (2002b), os tratamentos de Pinus caribeae variedades bahamensis, caribaea e hondurensis apresentavam para DAP, respectivamente 17,03 ; 20,02 e $23,07 \mathrm{~cm}$, altura de plantas 12,$99 ; 13,82$ e 14,66 e para sobrevivência 72,50; 52,50 e 47,50 \% e os tratamentos de Pinus oocarpa (Honduras) e Pinus oocarpa (Brasil) apresentavam respectivamente para DAP (22,96 e $27,12 \mathrm{~cm})$; altura de plantas $(15,27$ e $14,85 \mathrm{~m})$ e sobrevivência $(43,75$ e 26,25 $\%)$ condições mantidas até o presente estudo.

Silva et al. (2011b) e Moraes et al. (2007) em trabalhos realizados com Pinus caribaea var. caribaea, Pinus caribaea var. hondurensis aos 14 anos e Missio et al. (2004) com Pinus caribaea var. bahamensis aos 13 anos de idade, obtiveram em Latossolo Vermelho Distrófico não degradado valores para o caráter DAP 23,$75 ; 25,04$ e 20,63 $\mathrm{cm}$, altura de plantas 19,$47 ; 23,20$ e $18,85 \mathrm{~m} \mathrm{e}$ volume 0,$624 ; 0,750$ e $0,547 \mathrm{~m}^{3}$ arv $^{-1}$, possibilitando entender que as espécies de Pinus caribaea no presente trabalho possuem altura, diâmetro a altura do peito e volume menores, evidenciando o efeito do solo degradado sobre o desenvolvimento das plantas.

Houve diferenças significativas entre os tratamentos para fósforo, matéria orgânica, $\mathrm{pH}$, magnésio, hidrogênio mais alumínio, alumínio, soma de bases, capacidade de troca cationnica e saturação por bases e para as camadas estudadas não teve significância para $\mathrm{pH}$, potássio e cálcio (Tabela 2).

Os valores médios para fósforo entre os tratamentos de Pinus encontram-se muito abaixo em relação ao Pinus caribaea var. bahamensis $(6,25$ $\mathrm{mg} \mathrm{dm} \mathrm{d}^{-3}$ ) que apresentou o maior valor, enquanto a testemunha é o tratamento de menor conteúdo $\left(1,69 \mathrm{mg} \mathrm{dm}^{-3}\right)$. Há uma diminuição do fósforo com o aumento da profundidade do solo. $\mathrm{Na}$ camada de $0,00-0,10 \mathrm{~m}, \mathrm{o}$ fósforo encontra-se com conteúdo médio para essências florestais, que é de 6 a $8 \mathrm{mg}$ $\mathrm{dm}^{-3}$ (RAIJ et al., 1996).

O tratamento com Pinus caribaea var. hondurensis apresentou o maior teor de matéria orgânica, com $19 \mathrm{~g} \mathrm{dm}^{-3}$ (Tabela 2), diferindo estatisticamente do Pinus oocarpa (Honduras) com $12 \mathrm{~g} \mathrm{dm}^{-3}$. O Pinus caribaea depositou maior quantidade de matéria orgânica, provavelmente por possuir fascículo de acículas com bainha caduca com duração de 2 anos, enquanto que o Pinus

TABELA 1: Valores médios obtidos para as características silviculturais, envolvendo cinco espécies de Pinus, em Selvíria - MS.

TABLE 1: Average values for silvicultural traits, including five species of Pinus in Selvíria - MS state.

\begin{tabular}{lccccccc}
\hline \multirow{2}{*}{ Tratamentos } & \multicolumn{7}{c}{ Caracteres Silviculturais } \\
\cline { 2 - 8 } & $\begin{array}{c}\text { DAP } \\
(\mathrm{cm})\end{array}$ & $\begin{array}{c}\text { ALT } \\
(\mathrm{m})\end{array}$ & VOL $\left(\mathrm{m}^{3} \mathrm{arv}^{-1}\right)$ & ${ }^{1} \mathrm{FOR}$ & ${ }^{1} \mathrm{FT}$ & ${ }^{1} \mathrm{BIF}$ & ${ }^{1} \mathrm{SOB}(\%)$ \\
\hline Pinus caribaea var. bahamensis & $17,18 \mathrm{~b}$ & $13,75 \mathrm{a}$ & $0,32 \mathrm{~b}$ & $1,92 \mathrm{a}$ & $1,25 \mathrm{a}$ & $2,00 \mathrm{a}$ & $71,25 \mathrm{a}$ \\
Pinus caribaea var. caribaea & $20,23 \mathrm{~b}$ & $14,45 \mathrm{a}$ & $0,47 \mathrm{~b}$ & $2,17 \mathrm{a}$ & $1,23 \mathrm{a}$ & $1,98 \mathrm{a}$ & $52,50 \mathrm{ab}$ \\
Pinus caribaea var. hondurensis & $23,58 \mathrm{ab}$ & $16,00 \mathrm{a}$ & $0,71 \mathrm{ab}$ & $2,12 \mathrm{a}$ & $1,32 \mathrm{a}$ & $1,96 \mathrm{a}$ & $47,50 \mathrm{ab}$ \\
Pinus oocarpa (Honduras) & $22,93 \mathrm{ab}$ & $15,85 \mathrm{a}$ & $0,66 \mathrm{ab}$ & $1,92 \mathrm{a}$ & $1,22 \mathrm{a}$ & $1,90 \mathrm{a}$ & $41,25 \mathrm{ab}$ \\
Pinus oocarpa (Brasil) & $27,45 \mathrm{a}$ & $16,28 \mathrm{a}$ & $1,01 \mathrm{a}$ & $2,10 \mathrm{a}$ & $1,22 \mathrm{a}$ & $1,86 \mathrm{a}$ & $26,25 \mathrm{~b}$ \\
\hline Média & 22,27 & 15,27 & 0,63 & 2,05 & 1,25 & 1,94 & 47,75 \\
CV (\%) & 13,19 & 8,20 & 36,11 & 7,79 & 4,84 & 3,93 & 19,79 \\
F (TRAT) & 6,85 & 3,10 & 5,18 & 2,20 & 1,67 & 2,32 & 3,35 \\
Pr> F & 0,0041 & 0,0573 & 0,0116 & 0,1301 & 0,2213 & 0,1163 & 0,0463 \\
\hline
\end{tabular}

Em que: ${ }^{1}$ Para efeito de análise estatística os dados foram transformados em $\sqrt{x+0,5}$. DAP $=$ diâmetro a altura do peito; $\mathrm{ALT}=$ altura de plantas; $\mathrm{VOL}=$ volume; $\mathrm{FOR}=$ forma de fuste; $\mathrm{FT}=$ foxtail $; \mathrm{BIF}=$ bifurcação de plantas; $\mathrm{SOB}$ = sobrevivência de plantas. 
oocarpa possui fascículo de acículas com bainha persistentes.

Por serem espécies diferentes em relação à vegetação nativa dos cerrados, os Pinus possuem estruturas diferentes de copa, pois, a quantidade de espaço ocupado pelos troncos, galhos, ramos e acículas, proporcionam uma deposição diferenciada de material orgânico. No processo de recuperação das propriedades físicas, químicas e biológicas do solo, os efeitos da interação da quantidade e qualidade do material orgânico, das espécies que estão sendo utilizadas e das condições climáticas do local, são importantes para a compreensão dos resultados. Esses fatos podem promover diferentes efeitos na restauração das condições originais do solo, uma vez que as espécies diferem quanto à dinâmica do sistema radicular e às atividades biológicas no solo (CAVENAGE, 1996). No trabalho de Poggiani (1979), com Pinus oocarpa e Pinus caribaea var. hondurensis, a quantidade de nutrientes variou entre as duas espécies, visto que o Pinus caribaea var. hondurensis retorna ao solo uma quantidade maior de acículas e, consequentemente, uma maior quantidade de elementos químicos.

Cavenage (1996) encontrou para cerrado o teor $39 \mathrm{~g} \mathrm{dm}^{-3}$ de matéria orgânica na camada de $0,00-0,10 \mathrm{~m}$, valor acima dos encontrados neste trabalho e para Pinus caribaea var. hondurensis, o autor encontrou valor de $24 \mathrm{~g} \mathrm{dm}^{-3}$, tendo-se então que a deposição de matéria orgânica pelos Pinus estudados em área degradada é semelhante ao trabalho mencionado em solo que não houve remoção da camada superficial. Entre as camadas de estudo, verificou-se uma diminuição do teor de matéria orgânica com o aumento da profundidade do solo, conforme o esperado, por ser este o comportamento natural nos solos. As espécies de Pinus caribaea apresentaram os maiores valores para o teor de matéria orgânica, esta deposição de material orgânico provavelmente proporciona uma melhor cobertura do solo e dos atributos químicos, o que garante uma melhor sobrevivência de plantas em relação aos Pinus oocarpa.

Para os tratamentos e camadas estudadas, os valores médios de $\mathrm{pH}$ são considerados com acidez alta a muito alta $(4,3$ e 5,0$)$, segundo Raij et al. (1996). O Pinus oocarpa (Honduras) diferiu estatisticamente dos demais tratamentos $(4,6)$ (Tabela 2). Alves (2001), trabalhando com a utilização de calcário, gesso e adubos verde na recuperação de solo no mesmo local e condições de estudo, obteve resultados semelhantes, ou seja,
pH entre 4,5 a 5,0. Cavenage (1996), em Latossolo Vermelho-Escuro na mesma região de cerrado, encontrou resultados para $\mathrm{pH}$ entre 4,6 a 4,9, também semelhantes a este trabalho. Em termos de nutrição de plantas, a faixa ideal de $\mathrm{pH}$ situa-se em torno de 5,6 a 6,2. Nessa faixa, o alumínio encontrase precipitado e a maioria dos nutrientes encontrase em formas solúveis e, assim, passíveis de serem absorvidas pelas raízes. No entanto, com a evolução dos programas de levantamentos de espécies com potencial para utilização em recuperação de áreas degradadas, tem sido observada ampla gama de espécies que toleram valores de $\mathrm{pH}$ na faixa de 4,2 a 5,0. Essas plantas apresentam mecanismos internos e externos ao sistema radicular que fazem com que tolerem a acidez elevada, apesar da maior disponibilidade de elementos que possam causar fitotoxidez (DIAS, 1998). As espécies de Pinus demonstram-se tolerantes a acidez do solo em estudo, por apresentarem um bom desenvolvimento neste solo degradado. Barret e Golfari (1962) concluem que o Pinus caribaea var. caribaea tem uma faixa de $\mathrm{pH}$ ideal entre 4,5 e 6,0 ; o Pinus caribaea var. hondurensis entre 4,0 e 6,5 e Pinus caribaea var. bahamensis entre 7,5 e 8,5.

Não houve variação entre os tratamentos e camadas para os teores de cálcio e potássio estudados (Tabela 2). Segundo Raij et al. (1996), estes elementos se encontram baixo para potássio $\left(0,8\right.$ a $\left.1,5 \mathrm{mmol}_{\mathrm{c}} \mathrm{dm}^{-3}\right)$ e de médio a alto para cálcio (de 4 a maior que $7 \mathrm{mmol}_{\mathrm{c}} \mathrm{dm}^{-3}$ ).

A relação $\mathrm{K} / \mathrm{Ca}$ é sempre menor nos piores sítios, nos quais o potássio é deficiente. Nessas áreas de arenito, ambos os nutrientes são ofertados em quantidades irrisórias. A diferença é que os requerimentos de potássio são superiores àqueles do cálcio. No entanto, a importância do cálcio não deve ser desmerecida, uma vez que quantidades expressivas deste elemento são exportadas. Tudo leva a crer que o bom suprimento de potássio acha-se em torno ou acima de $6,0 \mathrm{~g} \mathrm{~kg}^{-1}$ da massa seca, nos quais, bons níveis de crescimento foram verificados entre $6,0-12,0 \mathrm{~g} \mathrm{~kg}^{-1}$ para Pinus de 8 anos de idade (REISSMANN, 1981; REISSMANN; WISNEWSKI, 2000). Os menores teores observados em povoamentos com 15 anos sugerem ser o efeito da idade, uma vez que nos solos mais férteis não foram observados teores mais elevados (REISSMANN; WISNEWSKI, 2000).

O tratamento com Pinus caribaea var. hondurensis apresentou valor médio de 4,44 $\mathrm{mmol}_{\mathrm{c}}$ $\mathrm{dm}^{-3}$ para o elemento magnésio (Tabela 2), diferindo 
TABELA 2: Quadrados médios, coeficientes de variação e valores médios de fósforo $(\mathrm{P})$, matéria orgânica $(\mathrm{MO}), \mathrm{pH}$, potássio $(\mathrm{K})$, cálcio $(\mathrm{Ca})$, magnésio $(\mathrm{Mg})$, hidrogênio $(\mathrm{H})+$ alumínio $(\mathrm{Al})$, alumínio (Al), soma de bases (SB), capacidade de troca catiônica (CTC) e saturação por bases (V), em função dos tratamentos e camadas estudadas em Selvíria - MS.

TABLE 2: Mean squares, coefficients of variation and average values of phosphorus $(\mathrm{P})$, organic matter $(\mathrm{MO}), \mathrm{pH}$, potassium $(\mathrm{K})$, calcium $(\mathrm{Ca})$, magnesium $(\mathrm{Mg})$, hydrogen $(\mathrm{H})+$ aluminum $(\mathrm{Al})$, aluminum (Al), total bases (SB), cation exchange capacity (CTC) and base saturation (V), depending on the treatments and depths studied in Selvíria - MS state.

\begin{tabular}{|c|c|c|c|c|c|c|c|c|c|c|c|}
\hline \multirow{2}{*}{ Tratamento } & $\mathrm{P}$ & $\mathrm{MO}$ & $\mathrm{pH}$ & $\mathrm{K}$ & $\mathrm{Ca}$ & $\mathrm{Mg}$ & $\mathrm{H}+\mathrm{Al}$ & $\mathrm{Al}$ & SB & CTC & $\mathrm{V}$ \\
\hline & mg.dm ${ }^{-3}$ & g.dm ${ }^{-3}$ & $\mathrm{CaCl}_{2}$ & \multicolumn{3}{|c|}{ 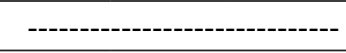 } & \multicolumn{4}{|c|}{ 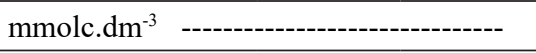 } & $\%$ \\
\hline Pcb & $6,25 \mathrm{a}$ & $16 \mathrm{ab}$ & $4,4 \mathrm{ab}$ & $1,45 \mathrm{a}$ & $8,62 \mathrm{a}$ & $4,06 \mathrm{a}$ & $31,50 \mathrm{ab}$ & $4,13 b c$ & $14,94 \mathrm{ab}$ & $46,44 \mathrm{ab}$ & $30,81 \mathrm{a}$ \\
\hline Pcc & $2,13 b$ & $15 \mathrm{abc}$ & $4,3 b$ & $1,20 \mathrm{a}$ & $8,00 \mathrm{a}$ & $3,38 \mathrm{ab}$ & $31,75 \mathrm{a}$ & $5,25 \mathrm{ab}$ & $12,60 \mathrm{ab}$ & $44,35 \mathrm{ab}$ & $26,75 \mathrm{a}$ \\
\hline Pch & $3,31 \mathrm{ab}$ & $19 \mathrm{a}$ & $4,3 b$ & $0,95 \mathrm{a}$ & $10,44 a$ & $4,44 \mathrm{a}$ & $33,56 \mathrm{a}$ & $4,38 \mathrm{abc}$ & $15,90 \mathrm{a}$ & $49,46 a$ & $30,50 \mathrm{a}$ \\
\hline $\begin{array}{l}\text { Po } \\
\text { (Honduras) }\end{array}$ & $2,06 \mathrm{~b}$ & $12 \mathrm{c}$ & $4,6 a$ & $0,93 \mathrm{a}$ & $8,63 a$ & $3,63 a b$ & $27,38 \mathrm{c}$ & $3,38 \mathrm{c}$ & $13,18 \mathrm{ab}$ & $40,55 \mathrm{bc}$ & $30,44 a$ \\
\hline Po (Brasil) & $2,31 b$ & $13 b c$ & $4,2 b$ & $1,06 \mathrm{a}$ & $5,38 \mathrm{a}$ & $3,06 \mathrm{ab}$ & $33,69 \mathrm{a}$ & $5,81 \mathrm{a}$ & $9,48 b$ & $43,17 b$ & $22,06 \mathrm{a}$ \\
\hline Testemunha & $1,69 b$ & $15 \mathrm{bc}$ & $4,2 b$ & $1,59 \mathrm{a}$ & $6,13 a$ & $2,50 \mathrm{~b}$ & $27,88 \mathrm{bc}$ & $4,88 \mathrm{ab}$ & $9,14 b$ & $37,02 \mathrm{c}$ & $23,31 \mathrm{a}$ \\
\hline $\begin{array}{l}\text { DMS-Tukey } \\
5 \%\end{array}$ & 3,53 & 3,70 & 0,25 & 1,71 & 5,42 & 1,54 & 3,74 & 1,47 & 6,05 & 5,99 & 9,47 \\
\hline F (Trat) & $46,37^{* *}$ & $84,29^{* *}$ & $0,30^{* *}$ & $1,19^{\mathrm{ns}}$ & $54,43^{\mathrm{ns}}$ & $7,74^{* *}$ & $119,99^{* *}$ & $11,96^{* *}$ & $122,67^{* *}$ & $306,31^{* *}$ & $243,38^{*}$ \\
\hline \multicolumn{12}{|l|}{ Camada (m) } \\
\hline $0,00-0,10$ & $6,38 \mathrm{a}$ & $24 \mathrm{a}$ & $4,4 a$ & $1,50 \mathrm{a}$ & $10,21 \mathrm{a}$ & $5,13 \mathrm{a}$ & $34,25 \mathrm{a}$ & $3,63 b$ & $16,70 \mathrm{a}$ & $50,95 \mathrm{a}$ & $31,79 a$ \\
\hline $0,10-0,20$ & $2,42 b$ & $13 b$ & $4,4 a$ & $1,14 \mathrm{a}$ & $7,16 \mathrm{a}$ & $3,13 b$ & $29,71 b$ & $4,46 a b$ & $12,07 \mathrm{~b}$ & $41,78 b$ & $27,67 \mathrm{a}$ \\
\hline $0,20-0,40$ & $1,50 \mathrm{~b}$ & $11 \mathrm{~b}$ & $4,3 \mathrm{a}$ & $1,45 \mathrm{a}$ & $6,38 \mathrm{a}$ & $3,42 b$ & $30,46 b$ & $5,33 \mathrm{a}$ & $10,60 \mathrm{~b}$ & $41,06 \mathrm{~b}$ & $24,88 \mathrm{a}$ \\
\hline $0,40-0,60$ & $1,54 b$ & $11 \mathrm{~b}$ & $4,3 \mathrm{a}$ & $0,69 \mathrm{a}$ & $7,71 \mathrm{a}$ & $2,38 b$ & $29,42 b$ & $5,13 \mathrm{a}$ & $10,79 b$ & $40,20 \mathrm{~b}$ & $24,92 \mathrm{a}$ \\
\hline $\begin{array}{l}\text { DMS-Tukey } \\
5 \%\end{array}$ & 2,58 & 2,70 & 0,17 & 1,25 & 3,97 & 1,13 & 2,74 & 1,08 & 4,43 & 4,38 & 6,93 \\
\hline F (Prof) & $128,81^{* *}$ & $930,45^{* *}$ & $0,066^{\mathrm{ns}}$ & $3,37^{\mathrm{ns}}$ & $65,83^{\mathrm{ns}}$ & $32,43^{* *}$ & $120,19^{* *}$ & $14,23^{* *}$ & $194,88^{* *}$ & $602,30^{* *}$ & $254,96^{*}$ \\
\hline $\begin{array}{l}F \text { (Trat } x \\
\text { Prof) }\end{array}$ & $21,54^{*}$ & $12,19^{\mathrm{ns}}$ & $0,12^{*}$ & $2,92^{\mathrm{ns}}$ & $36,12^{\text {ns }}$ & $4,85^{*}$ & $22,15^{\text {ns }}$ & $4,53^{*}$ & $36,10^{\text {ns }}$ & $46,30^{\text {ns }}$ & $86,93^{\text {ns }}$ \\
\hline $\mathrm{CV} \%$ & 113,31 & 23,46 & 5,56 & 135,95 & 65,53 & 41,79 & 11,48 & 30,19 & 45,83 & 13,08 & 32,95 \\
\hline
\end{tabular}

Em que: Médias, seguidas de mesma letra na coluna, para cada fator, não diferem estatisticamente entre si, no nível de $5 \%$ de probabilidade pelo teste de Tukey. ${ }^{* *} ;{ }^{*} \mathrm{e}^{\text {ns }}$ são, respectivamente, significativo a $1 \%, 5 \%$ e não significativo no teste F. Pcb = Pinus caribaea var. bahamensis; Pcc = Pinus caribaea var. caribaea Pch = Pinus caribaea var. hondurensis; Po $=$ Pinus oocarpa .

estatisticamente somente da testemunha, que apresentou o menor valor $\left(2,50 \mathrm{mmol}_{\mathrm{c}} \mathrm{dm}^{-3}\right)$. Alves (2001), estudando a recuperação deste mesmo solo com adubação química e adubos verdes, encontrou valores baixos para o magnésio, demonstrando esta área ser deficiente neste nutriente. Para as camadas estudadas, houve uma diminuição de magnésio da camada superficial para as demais camadas. Quantidades maiores de magnésio encontram-se na camada de $0,00-0,10 \mathrm{~m}\left(5,15 \mathrm{mmol}_{\mathrm{c}} \mathrm{dm}^{-3}\right)$. Os valores encontrados para os tratamentos enquadramse na classificação de Raij et al. (1996) como baixo e médio para a camada de $0,00-0,10 \mathrm{~m}(0-4$ baixo; $5-8 \mathrm{mmol}_{\mathrm{c}} \mathrm{dm}^{-3}$ - médio). Evidenciou-se que, no mínimo, 0,6 $\mathrm{g} \mathrm{kg}^{-1}$ de magnésio são necessários para um adequado crescimento do Pinus, valor encontrado nas melhores classes de sítio (REISSMANN; WISNEWSKI, 2000). A área de cerrado estudada por Cavenage (1996) apresentou os seguintes valores na camada de $0,00-0,10 \mathrm{~m}: 2,30 ; 30,40$; $10,10 \mathrm{mmol}_{\mathrm{c}} \mathrm{kg}^{-1}$, para, respectivamente, potássio, cálcio e magnésio. A área de estudo apresenta-se com baixos teores destes nutrientes em relação ao cerrado.

Apesar de haver um acúmulo de nutrientes na superfície do solo, em florestas implantadas de Eucalyptus e Pinus representado pela manta, a ação destes nas características químicas do solo se faz 
praticamente apenas no aumento do teor de matéria orgânica (HAAG, 1983).

Para os teores de hidrogênio mais alumínio houve significância estatística entre os tratamentos e camadas (Tabela 2). Os Pinus caribaea var. bahamensis, Pinus caribaea var. caribaea, Pinus caribaea var. hondurensis e Pinus oocarpa (Brasil) não diferiram estatisticamente entre si. Enquanto que o menor valor encontrado para estes elementos foi do Pinus oocarpa (Honduras) com 27,38 $\mathrm{mmol}_{\mathrm{c}} \mathrm{dm}^{-3}$ não diferindo da testemunha. Alves (2001) encontrou resultados menores neste solo, estando entre 15,00 a $17,04 \mathrm{mmol}_{\mathrm{c}} \mathrm{dm}^{-3}$, devido aos tratamentos que receberam calagem e calagem + gesso, com maior influência na camada de 0,00 $-0,10 \mathrm{~m}$. Para as camadas estudadas, a camada de $0,00-0,10 \mathrm{~m}$ apresenta maior valor de hidrogênio mais alumínio $\left(34,25 \mathrm{mmol}_{\mathrm{c}} \mathrm{dm}^{-3}\right)$ em relação às demais camadas. Estes resultados são contrários aos de Alves (2001), nos quais o menor valor foi encontrado na camada superficial $\left(14,98 \mathrm{mmol}_{\mathrm{c}}\right.$ $\left.\mathrm{dm}^{-3}\right)$. Resultados semelhantes ao presente trabalho foram encontrados por Cavenage (1996) na camada de $0,00-0,10 \mathrm{~m}$ para área de mata ciliar, cerrado e Pinus caribaea var hondurensis (respectivamente 39,$10 ; 38,00$ e $35,40 \mathrm{mmol}_{\mathrm{c}} \mathrm{kg}^{-1}$ ).

O Pinus oocarpa (Honduras e Brasil) apresentaram o menor e o maior valor para o alumínio (respectivamente, 3,38 e 5,81 $\mathrm{mmol}_{\mathrm{c}} \mathrm{dm}^{-3}$ ) (Tabela 2). Para este elemento houve significância para os tratamentos e camadas, em que, o menor valor de alumínio encontra-se na camada de $0,00-0,10(3,63$ $\mathrm{mmol}_{\mathrm{c}} \mathrm{dm}^{-3}$ ), e com o aumento da profundidade há um aumento de alumínio, tendência também encontrada por Alves (2001) e Cavenage (1996). $\mathrm{O}$ último autor encontrou esta tendência ocorrendo naturalmente em mata ciliar e cerrado e também concluiu uma relação existente entre o alumínio e o $\mathrm{pH}$ de forma negativa para a área de eucalipto, pois, quanto menor o $\mathrm{pH}$ maior a quantidade de alumínio e vice-versa, e isto também ocorreu nos tratamentos de Pinus oocarpa (Honduras e Brasil) estudados, nos quais tem-se o menor $\mathrm{pH}$ e a maior quantidade de alumínio e vice-versa. Alves (2001), neste mesmo solo com o uso de calagem, adubos verdes e gesso, encontrou valores de alumínio entre 1,00 e 1,56 mmol $\mathrm{dm}^{-3}$ para os tratamentos estudados. Veiga et al. (1977), estudando povoamentos de Pinus elliottii var. elliottii em diferentes localidades do Estado de São Paulo, visando conhecer as suas respostas ante a deficiência natural de macronutrientes e a limites intoleráveis de alumínio, concluíram que o limite de tolerância ao alumínio parece ser maior que 5 , talvez, em torno de 25 ou $30 \mathrm{mmol}_{\mathrm{c}} \mathrm{dm}^{-3}$ de solo.

$\mathrm{O}$ tratamento com maior valor de soma de bases e capacidade de troca cationica é o Pinus caribaea var. hondurensis, e os menores valores encontrados foram os da testemunha (Tabela 2). Para as camadas estudadas, a camada de $0,00-0,10 \mathrm{~m}$ difere estatisticamente das demais, pois, apresentou maiores valores de soma de bases e capacidade de troca catiônica (respectivamente, 16,70 e 50,95 $\mathrm{mmol}_{\mathrm{c}} \mathrm{dm}^{-3}$ ). Portanto, a cobertura residual do solo está contribuindo para aumento de nutrientes na camada superficial, melhorando as condições deste solo. Lopes (1984) classificou a capacidade de troca cationnica dos solos de cerrado como extremamente baixa. Em seu trabalho, $84 \%$ das amostras de solos analisadas apresentaram $11 \mathrm{mmol}_{\mathrm{c}} \mathrm{dm}^{-3}$.

A saturação por bases ( $\mathrm{V} \%$ ) não apresentou variação tanto para tratamentos como para as camadas (Tabela 2). Pela classificação de Raij et al. (1996), os resultados se enquadram com valores muito baixo a baixo (0-25 e 26-50\%). Se analisadas com os valores de Cavenage (1996) para área de cerrado (41,9 a 54,3\%), os resultados aqui encontrados estão baixos.

Na Tabela 3 são apresentadas espécies que se desenvolveram naturalmente no sub-bosque do experimento. Foram identificadas um total de 16 famílias e 18 espécies. As famílias Anacardiaceae e Malvaceae apresentaram maior diversidade com duas espécies e as demais famílias uma espécie cada.

Estudos de regeneração natural na região de Selvíria - MS em Latossolo Vermelho Distrófico não decapitado, os autores encontraram sob sistema silvipastoril de Myracrodruon urundeuva (GUERRA et al., 2008) 36 famílias e 71 espécies. Silva et al. (2011a), estudando os aspectos quantitativo e qualitativo da regeneração natural sob reflorestamento ciliar em dois modelos de plantio identificaram 33 famílias e 63 espécies. E em sub-bosque de Eucalyptus camaldulensis foram identificadas 74 espécies por Silva et al. (2011c).

As espécies nativas de cerrado têm potencial de regeneração em condições de solo degradado, apresentando alta diversidade de espécies, quando já existe uma cobertura vegetal de espécies nativas ou exóticas, como apresentado neste estudo.

Kageyama e Patiño (1985) citam autores que encontraram espécies arbóreas em ecossistemas de coníferas em diferentes regiões: Spurr e Barnes (1982) em Michigan, EUA (10 espécies); Valdez e 
Aguilar (1983), Nuevo Léon, México (28 espécies) e em floresta de Pinus guercus 64 espécies encontradas por estes últimos autores. Verificando que as espécies de Pinus realmente contribuem de forma positiva para a regeneração natural de solos degradados ou não.

As espécies que se desenvolveram no experimento possuem potencial de recuperação de áreas degradadas por estarem ocorrendo naturalmente neste local. Isto demonstra que, quando há uma cobertura do solo, todo o conjunto do ecossistema degradado tende a se regenerar, pois foi oferecida condição inicial para que este sistema começasse a recuperar-se. Trabalho realizado em

TABELA 3: Nome das espécies que estão ocorrendo naturalmente em consórcio com os Pinus.

TABLE 3: Name of species that are naturally occurring in consortium with Pinus.

\begin{tabular}{|c|c|}
\hline Família/Espécies & Nome vulgar \\
\hline \multicolumn{2}{|l|}{ ANACARDIACEAE } \\
\hline Astronium fraxinifolium Schott. ex Spreng. & gonçalo-alves \\
\hline Myracrodruon urundeuva Allemão & aroeira \\
\hline \multicolumn{2}{|l|}{ BIGNONIACEAE } \\
\hline Handroanthus spp. & ipê \\
\hline \multicolumn{2}{|l|}{ CANNABACEAE } \\
\hline Trema micrantha (L.) Blume & grão-de-galo \\
\hline \multicolumn{2}{|l|}{ COMBRETACEAE } \\
\hline Terminalia argentea Mart. & capitão-do-campo \\
\hline \multicolumn{2}{|l|}{ DILLENIACEAE } \\
\hline Curatella americana $\mathrm{L}$. & lixeira \\
\hline \multicolumn{2}{|l|}{ LEGUMINOSAE-CAESALPINOIDEAE } \\
\hline Peltophorum dubium (Spreng.) Taubert & canafístula \\
\hline \multicolumn{2}{|l|}{ LEGUMINOSAE-FABOIDEAE } \\
\hline Machaerium acutifolium Vog. & sapuva \\
\hline \multicolumn{2}{|l|}{ LEGUMINOSAE-PAPILIONOIDEAE } \\
\hline Acosmium sp. & calunga \\
\hline \multicolumn{2}{|l|}{ MALPIGHIACEAE } \\
\hline Byrsonima sp. & murici \\
\hline \multicolumn{2}{|l|}{ MALVACEAE } \\
\hline Luehea divaricata Mart. & açoita-cavalo \\
\hline Guazuma ulmifolia Lam. & mutambo \\
\hline \multicolumn{2}{|l|}{ MELIACEAE } \\
\hline Melia azedarach L. & santa-barbara \\
\hline \multicolumn{2}{|l|}{ MORACEAE } \\
\hline Maclura tinctoria (L.) D. Don ex Steud. & amora-branca \\
\hline \multicolumn{2}{|l|}{ MYRTACEAE } \\
\hline Psidium guajava L. & goiaba \\
\hline \multicolumn{2}{|l|}{ POLYGONACEAE } \\
\hline Coccoloba mollis Casar. & quina-doce \\
\hline \multicolumn{2}{|l|}{ RHAMNACEAE } \\
\hline Rhamnidium elaeocarpus Reissek & cafezinho \\
\hline \multicolumn{2}{|l|}{ RUBIACEAE } \\
\hline Alibertia edulis Rich. & marmelo \\
\hline
\end{tabular}


área ripária de região de Cerrado para verificar se o plantio de Pinus elliottii influencia na regeneração nativa em vários espaçamentos, Modna, Durigan e Vital (2010) concluíram que até onze anos após o plantio, as árvores de Pinus exerceram ação facilitadora da regeneração de espécies nativas em seu sub-bosque, sendo maior a densidade de plantas em regeneração quanto maior a biomassa de Pinus. Modna, Durigan e Vital (2010) em plantio de Pinus elliottii observaram 59 espécies lenhosas e destas, 21 espécies sob as copas do Pinus e em média foram amostrados 4.398 ind ha' ${ }^{-1}$.

O papel ou função que a espécie arbórea exerce na melhoria das condições de uma área degradada nos aspectos físico, químico e biológico do solo são mais importantes que a procedência da espécie utilizada, pois cada local a se recuperar tem uma situação própria. Informações a respeito do local a ser recuperado são de extrema importância, para um planejamento sustentável do solo. Tanto para florestas com fins de produção de matériaprima, como para áreas de preservação permanente em que se deseja recompor a floresta natural, isto, a longo prazo, para que haja interação floresta e solo.

\section{CONCLUSÕES}

A utilização de espécies de Pinus na recuperação de áreas degradadas, conhecidas como áreas de empréstimo, mostra-se promissora, destacando-se o Pinus caribaea var. hondurensis por apresentar um bom crescimento aliado a sua sobrevivência, o que garante a cobertura do solo.

As espécies de Pinus contribuíram para a melhoria das condições químicas do solo na camada $0,00-0,10 \mathrm{~m}$, devido à deposição de matéria orgânica, destacando o Pinus caribaea var. hondurensis por apresentar maior deposição desta, aumentando a matéria orgânica, soma de bases e capacidade de troca catiônica.

O Pinus caribaea var bahamensis é o mais eficaz na ciclagem do fósforo no solo.

Os Pinus destacam-se em relação à testemunha (condição natural), por cobrir rapidamente o solo degradado, fornecendo condições para que a sucessão da vegetação ocorra, servindo de abrigo para a fauna e principalmente por formar uma camada orgânica na qual as sementes e microorganismos começam a atuar mais intensamente na melhoria da estrutura do solo.

As espécies de Pinus estão proporcionando condições para o aumento da diversidade de espécies espontâneas.

\section{REFERÊNCIAS BIBLIOGRÁFICAS}

ALVES, M. C. Recuperação do subsolo de um Latossolo Vermelho usado para terrapleno e fundação da Usina Hidrelétrica de Ilha Solteira - SP. 2001. 83 f. Tese (Concurso de Livre-docência em Solos) - Universidade Estadual Paulista, Ilha Solteira, 2001.

ANGIOSPERM PHYLOGENY GROUP II. Na update of the Angiosperm Phylogeny Group classification for the orders and families of flowering plants. Botanical Journal of the Linnean Society, Oxford, v. 141, n. 4, p. 399-436, 2003.

BARRET, W.; GOLFARI, L. H. G. Descripción de dos nuevas variedades del "Pino del Caribe" (Pinus caribaea Morelet.). Caribbean Forester, Porto Rico, v. 23, n. 2, p. 59-71, 1962.

CAMPELLO, E. F. C. Sucessão vegetal na recuperação de áreas degradadas. In: DIAS, L. E.; MELLO, J. W. V. de (Ed.). Recuperação de áreas degradadas. Viçosa: Universidade Federal de Viçosa, Departamento de Solos, 1998. p. 183-196.

CAVENAGE, A. Alterações das propriedades físicas e químicas de um Latossolo Vermelho Escuro sob diferentes usos e manejos. 1996. 75 f. (Trabalho de Graduação) - Universidade Estadual Paulista, Ilha Solteira, 1996.

CENTURION, J. F. Balanço hídrico na região de Ilha Solteira. Científica, Jaboticabal, v. 10, n. 1, p. 57-61, 1982.

DEMATTÊ, J. L. I. Levantamento detalhado dos solos do campus experimental de Ilha Solteira. Piracicaba: ESALQ/USP, 1980. 131 p.

DIAS, L. E. Caracterização de substratos para fins de recuperação de áreas degradadas. In: DIAS, L. E.; MELLO, J. W. V. (Ed.). Recuperação de áreas degradadas. Viçosa: Universidade Federal de Viçosa, Departamento de Solos, 1998. p. 183-196.

GUERRA, C. R. S. B. et al. Levantamento de espécies florestais em área de sistema silvipastoril. Ciências Agrárias e Saúde, Andradina, v. 8, p. 40-45, 2008.

GURGEL FILHO, O. A. Silvicultura e economia de Pinus no Estado de São Paulo. Silvicultura em São Paulo, São Paulo, v. 4-5, n. 4, p. 209-234, 1965-1966.

HAAG, H. P. (Coord.). Nutrição mineral de Eucalyptus, Pinus, Araucaria e Gmelina no Brasil. Campinas: Fundação Cargill, 1983. 101 p.

INATOMI, T. A. H.; UDAETA, M. E. M. Análise 
dos impactos ambientais na produção de energia dentro do planejamento integrado de recursos. Seção de estudos estratégicos de energia e de desenvolvimento sustentável do GEPEA/ EPUSP. 2011. Disponível em: <http://seeds.usp. br/portal/uploads/INATOMI_TAHI_IMPACTOS AMBIENTAIS.pdf. Acesso em: $201 \overline{1}$.

KAGEYAMA, P. Y.; PATIÑO-VALERA, F. Conservación y manejo de recursos genéticos forestales: factores que influyen en la estructura y diversidad de los ecosistemas forestales. Documento Especial. In: CONGRESSO FORESTAL MUNDIAL, 9., 1985, México. Anais... 1985.

KAGEYAMA, P. Y.; PIRES, I. E.; HERRERA PRERA, L. E. Phenotypic and genotypic variations for foxtail growth in Pinus caribaea var. hondurensis. In: PROCEEDINGS OF A JOINT CONFERENCE OF IUFRO, 1984, Mutare, Zimbabwe. Anais... 1984. p. 526-534.

LOPES, A. S. "Solos sob cerrados": características, propriedades e manejo. Piracicaba: Instituto da Potassa \& Fosfato, 1984. 162 p.

LORENZI, H. Árvores brasileiras: manual de identificação e cultivo de plantas arbóreas nativas do Brasil. 2. ed. Nova Odessa, SP: Plantarum, 2002. v. 2.

LORENZI, H. Árvores brasileiras: manual de identificação e cultivo de plantas arbóreas nativas do Brasil. 5. ed. Nova Odessa, SP: Plantarum, 2008. V. 1.

LORENZI, H. Árvores brasileiras: manual de identificação e cultivo de plantas arbóreas nativas do Brasil. 1. ed. Nova Odessa, SP: Plantarum, 2009. v. 3.

MECHI, A.; SANCHES, D. L. Impactos ambientais da mineração no Estado de São Paulo. Estudos Avançados, São Paulo, v. 24, n. 68, p. 209-220, 2010.

MISSIO, R. F. et al. Seleção simultânea de caracteres em progênies de Pinus caribaea Morelet var. bahamensis. Scientia Forestalis, Piracicaba, n. 66, p. 161-168, 2004.

MODNA, D.; DURIGAN, G.; VITAL, M. V. C. Pinus elliottii Engelm como facilitadora da regeneração natural da mata ciliar em região de Cerrado, Assis, SP, Brasil. Scientia Forestalis, Piracicaba, v. 38, n. 85, p. 73-83, 2010.

MORAES, M. L. T. et al. Efeito do desbaste seletivo nas estimativas de parâmetros genéticos em progênies de Pinus caribaea Morelet var. hondurensis. Scientia Forestalis, Piracicaba, n. 74, p. $55-65,2007$.
MÜLLER, A. C. Hidrelétricas, meio ambiente e desenvolvimento. São Paulo: Makron Books, 1995. $412 \mathrm{p}$.

NOFFS, P. S. Informações geomorfológicas para reflorestamento de essências nativas. In: CONGRESSO NACIONAL SOBRE ESSÊNCIAS NATIVAS, 1982, Campos do Jordão, SP. Anais... 1982. p. 976-980.

POGGIANI, F. Deposição mensal de acículas e nutrientes em plantações homogêneas de Pinus oocarpa e Pinus caribaea var. hondurensis. Boletim Informativo PPT, Piracicaba, v. 7, p. 12-19, 1979. RAIJ, B. V. et al. (Eds.). Análise química para avaliação da fertilidade de solos tropicais. Campinas: Instituto Agronômico, 2001. 285 p.

RAIJ, B. V. et al. Recomendações de adubação e calagem para o Estado de São Paulo. 2. ed. Campinas: Instituto Agronômico \& Fundação IAC, 1996. 285 p. (Boletim técnico, 100).

REISSMANN, C. B. Naehrelementversorgung und Wuchsleistung von Kiefernbestaenden in Sued-Brasilien. 1981. 169 f. Tese (Doutorado em Ciências Florestais) - Forstwissenschaftlichen Fakultät der Albert-Ludwigs-Universitat Freiburg, Freiburg, 1981.

REISSMANN, C. B.; WISNEWSKI, C. Aspectos nutricionais de plantios de Pinus. In: GONÇALVES, J. L. M.; BENEDETTI, V. Nutrição e fertilização florestal. Piracicaba: IPEF, 2000. p. 135-165.

SANTOS, H. G. et al. Sistema Brasileiro de Classificação de Solos. 3. ed. Brasília, DF: Embrapa, 2013. 353 p.

SAS INSTITUTE. SAS/STAT User's Guide: version 6. 4. ed. Cary: SAS institute, 1990. v. 2.

SILVA, A. M. et al. Recuperação de áreas degradadas com a utilização de diferentes espécies de Pinus ssp e manejo do solo, em Selvíria - MS. In: SIMPÓSIO NACIONAL SOBRE RECUPERAÇÃO DE ÁREAS DEGRADADAS, Água e Biodiversidade, 5., 2002, Belo Horizonte. Anais... Belo Horizonte: Sociedade Brasileira de Recuperação de Áreas Degradadas, 2002a. p. 451-453.

SILVA, A. M. et al. Recuperação de áreas degradadas com a utilização de diferentes espécies e variedades de Pinus. Cultura Agronômica, Ilha Solteira, v. 11, n. 1, p. 1-11, 2002 b.

SILVA, A. M. et al. Aspectos quantitativo e qualitativo da regeneração natural sob reflorestamento ciliar em dois modelos de plantio. Cultura Agronômica, Ilha Solteira, v. 20, n. 1, p. 51-70, 2011a.

SILVA, A. M. et al. Avaliação das propriedades 
químicas em solo de cerrado sob reflorestamento ciliar. Floresta, Curitiba, v. 42, n. 1, p. 49-58, 2012. SILVA, A. M.; MORAES, M. L. T.; BUZETTI, S. Propriedades químicas de solo sob reflorestamento ciliar após 20 anos de plantio em área de cerrado. Revista Árvore, Viçosa-MG, v. 35, n. 1, p. 97-106, 2011.

SILVA, J. M. et al. Variação genética e ganho esperado na seleção de progênies de Pinus caribaea var. caribaea em Selvíria, MS. Scientia Forestalis, Piracicaba, v. 39, n. 90, p. 241-251, 2011 b.
SILVA, J. M. et al. Regeneração do cerrado em sub-bosque de Eucalyptus camaldulensis Dehnh na região de Selvíria-MS. Cultura Agronômica, Ilha Solteira, v. 20, n. 2, p. 67-80, 2011c. SILVA JUNIOR, M. C. et al. 100 árvores do cerrado: guia de campo. Brasília: Rede de Sementes do Cerrado, 2005. 278 p.

VEIGA, A. A. et al. Macronutrientes e alumínio em povoamentos de Pinus elliottii var. elliottii. Boletim técnico Instituto Florestal, São Paulo, n. 27, p. 1-13, 1977. 